Ind. Health, 1964, 2, 95

\title{
STUDIES ON HAND PROTECTORS FOR PORTABLE VIBRATING TOOLS
}

\author{
I. MEASUREMENTS OF THE ATTENUATION EFFECT \\ OF POROUS ELASTIC MATERIALS
}

\author{
Toshisuke MIWA \\ National Institute of Industrial Health, Kizuki-Sumiyoshi, Kawasaki
}

(Received April 14, 1964)

\begin{abstract}
The protecting effect of the porous elastic materials inserted between the hand and the vibration source was examined by using the driving point mechanical impedance. This paper reports the results obtained with two types of polyvinyl chloride foam, whose shape was the cylinder of $5 \mathrm{~cm}$ diameter. One of these two types was found to be available above $30 \mathrm{c} / \mathrm{s}$ as the vibration protector.

The measuring method of this damping effect was also examined with the mechanical model of the hand.
\end{abstract}

It has been known that workers who use the pneumatic tools for long time are subject to circulatory disturbances of the hands. Not a few medical papers have dealt with this problem1). Although elastic materials inserted between the workers' hands and the vibrating tools have been empirically used for absorption of the vibration, the efficacy of the devices has not been analyzed theoretically.

The author tried to estimate the protective effect of the porous elastic materials on the compressive vibration correctly and to develop well-designed hand protectors. For this purpose, the attenuation effect was determined from the ratio of the driving point impedance of the human hand to that of the porous sample, which was pressed by the hand at the opposite side of the driving point. This ratio corresponds with that of the vibrational displacements at the both ends of the elastic cylindrical samples. The frequency range in this experiment was limited from $20 \mathrm{c} / \mathrm{s}$ to $500 \mathrm{c} / \mathrm{s}$.

The results were obtained with two types of polyvinyl chloride foam with completely closed cellular holes.

\section{PRINCIPLE OF VIBRATION DAMPING}

To quantify the protective effects of the porous elastic materials inserted between the hands and the vibration sources such as pneumatic tools, it is convenient to replace the hand and the excitor system by an analogous mechanical circuit. 


\section{T. MIWA}

Fig. 1 shows the equivalent mechanical circuit of this system, where the mass of the porous samples is negligibly small. In this case the vibrational component is assumed to be only compressive. The vector expression of the symbols indicates the complex quantities.

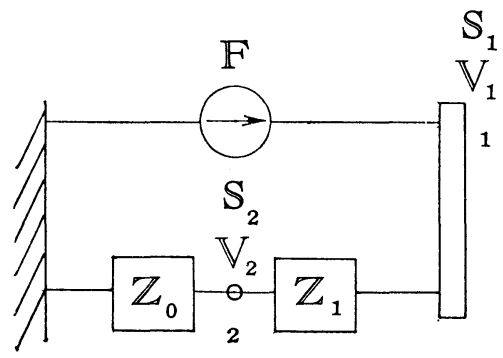

Fig. 1. Equivalent mechanical circuits of the case inserting the porous elastic material between the human hand and the vibration source.

FF ; a vibration force vector,

$\mathbb{V}:$ a vector of the vibration velocity due to the vibration force,

$S ;$ a vector of the vibration displacement due to the vibration force,

$Z_{0}$; the driving point mechanical impedance of the hand,

$\mathbb{Z}_{1}$; the driving point mechanical impedance of the porous elastic materials shunted with the rigid wall at the opposite side of the driving point. The subscripts 1 and 2 in the symbol $\mathbb{V}$ and $\mathbb{S}$ indicate their values at the points 1 and 2 in Fig. 1 resp. The vector expression of the symbols indicates the complex quantities.

For convenience, the author defined the attenuation of the vibration displacement in decibel unit $(\mathrm{dB})$;

$$
20 \log _{10}\left|S_{1} / S_{2}\right|
$$

where $S$ is $a$ vector of displacement due to the vibration force in Fig. 1 . The subscripts 1 and 2 in the displacement $S$ indicate their values at the points 1 and 2 respectively.

Hence, by using the equivalent circuit of Fig. 1, the following relation is obtained ;

$$
\left|\frac{\mathbb{S}_{1}}{\mathbb{S}_{2}}\right|=\left|\frac{\mathbb{V}_{1}}{\mathbb{V}_{2}}\right|=\left|\frac{Z_{0}}{\mathbb{Z}}\right|
$$

where $\mathbb{Z}=\frac{Z_{0} \cdot Z_{1}}{Z_{0}+Z_{1}}, \mathbb{V}_{1}, \mathbb{V}_{2}$ are vectors of vibration velocity due to the vibration force at the points of 1 and $2, Z_{0}$ is the driving point mechanical impedance of the hand, and $Z_{1}$ is the driving point mechanical impedance of the porous elastic material contacted with the rigid wall at the opposite side of the driving point.

The equation 2 implies that the displacement ratio at both ends of the sample is given as the ratio of the driving point mechanical impedance of the hand to that of the elastic material, which is pressed by the hand at the opposite side of driving 


\section{STUDIES ON HAND PROTECTORS I.}

point. The value of the positive $\mathrm{dB}$ indicates the efficacy of the materials. Therefore, the protective effect of the porous samples can be derived from the mechanical impedance.

\section{Apparatus and Principle of Measuring the Mechanical ImpedancE ${ }^{21}$}

The definition of the mechanical impedance is as follows:

$$
\mathbb{Z}=\frac{\mathbb{F}}{\mathbb{V}}=\frac{\mathbb{F}}{\mathrm{A}} j \omega=j \omega \alpha \mathbb{H}
$$

where $\mathrm{F}$ is the vibration force vector, $\mathrm{A}$ is a vector of the vibration acceleration due to the vibration force, $V$ is a vector of the vibration velocity due to the vibration force, $\omega$ is an angular frequency ( $2 \pi \times$ frequency), $\alpha$ is a magnification factor, and $H$ is the ratio of the output voltage of the force pickup to that of the acceleration pickup.

To measure the mechanical impedance under compressive vibration the apparatus was designed as shown in Fig. 2. The aluminium disk, which had a diameter of $5.0 \mathrm{~cm}$. and a thickness of $1.0 \mathrm{~cm}$, was fixed to the upper side of the force pickup and the vibration table of the excitor was fixed to the another side and the acceleration pickup was fixed on this disk. In the measurement of the impedance of the hand, the alminium disk had to be compressed by the hand with the constant force. Hence, the strain gauges were applied on the leaf spring, supporting the driver coil of the excitor, in order to watch this static pressure. The equivalent circuit of this system is presented in Fig. 3, (A). In this figure, the spring constant of the force and acceleration pickups $\left(k_{01}\right.$ and $\left.k_{02}\right)$ are so large that they are neglected in the following calculation. The mass of the pickup is devided into two parts by the sensing element. Then;



Fig. 2. Apparatus for measuring driving point mechanical impedance under compressive vibration. 
(A)

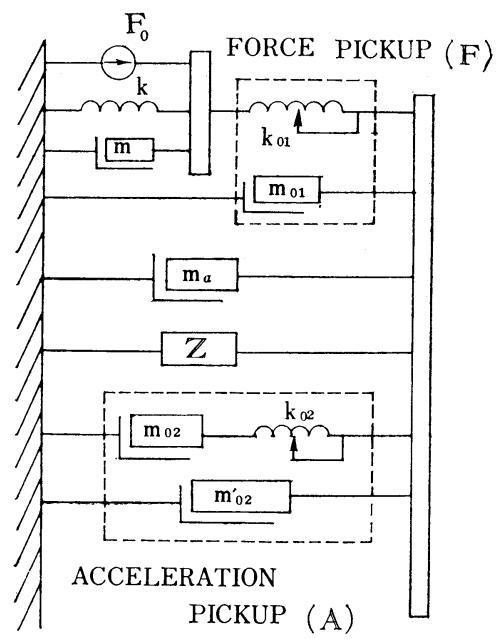

(B)

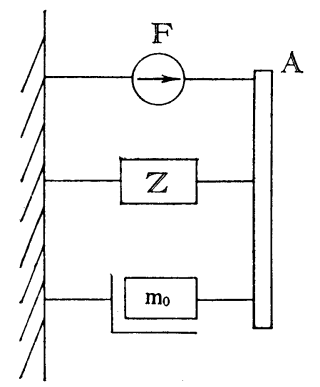

Fig. 3. (A) Equivalent mechanical circuit of measuring driving point mechanical impedance.

(B) Equivalent mechanical circuit simplified from Fig. 3 (A).

F ; a force observed with the force pickup.

$F_{0}$ : a force occured in the excitor,

$m$; the mass of the moving part of the driver coil of the excitor,

$k$; the spring constant of the leaf spring supporting the driver coil of the excitor,

$m_{01}, k_{01}$; the mass of the upper part from the sensing element and the spring constant of the force pickup,

$m_{\mathrm{a}}$; the mass of the aluminium disk,

$\mathbb{Z}$; an unknown mechanical impedance,

$m_{02}, m_{02}^{\prime}, k_{02}$ : masses of the upper and the lower part from the sensing element and the spring constant of the acceleration pickup respectively.

$\mathbb{A}$; an acceleration ofserved with the acceleration pickup,

As the force being impressed into the mechanical system was measured with the force pickup, the part consisted of $\mathbb{F}_{0}, \mathrm{k}$ and $\mathrm{m}$ as shown in Fig.3(A). can be neglected

$$
m_{0}=m_{01}+m_{02}+m_{02}{ }^{\prime}+m_{a}
$$

where $m_{01}$ is the mass of the force pickup in the upper part from the sensing element, $m_{02}, m_{02}{ }^{\prime}$ are masses of the acceleration pickup in the upper and the lower parts from the sensing element, respectively, and $m_{a}$ is the mass of the aluminium disk. Thus, Fig. 3 (A) is simplified as shown in Fig. 3 (B).

The magnification factor $\alpha$ in Eq. 3 and the additional mass of the measuring system $m_{0}$ in Eq. 4 should be determined to estimate the mechanical impedance. For this purpose, $\left|\mathbb{H}_{1}\right|,\left|\mathbb{H}_{2}\right|$ of two masses of different weights $\left(m_{1}, m_{2}\right)$ were measured. In this case, the equations are;

$$
\begin{aligned}
& j \omega\left(m_{1}+m_{0}\right)=j \omega \alpha\left|\mathbb{H}_{1}\right| \\
& j \omega\left(m_{2}+m_{0}\right)=j \omega \alpha\left|\mathbb{H}_{2}\right|
\end{aligned}
$$

The quantity $H$ becomes the real number in the example of the pure mass.

Thus, the equations of $\alpha$ and $m_{0}$ are; 


\section{STUDIES ON HAND PROTECTORS I.}

$$
\begin{aligned}
& \alpha=\left(m_{2}-m_{1}\right) /\left(H_{2}-H_{1}\right) \\
& m_{0}=\left(m_{2} H_{1}-m_{1} H_{2}\right) /\left(H_{2}-H_{1}\right)
\end{aligned}
$$

Therefore, the mechanical impedance of the practical material is obtained;

$$
\mathbb{Z}=j \omega\left[\left(\alpha \mathscr{R}(\mathbb{E})-m_{0}\right)-j \alpha \mathscr{F}(\mathbb{H}()]\right.
$$

where $\mathscr{R}$ shows the real part of the complex quantity $H$ and $\mathscr{F}$ the imaginary part of it.

It should be noticed that the mechanical impedance of the human hand is influenced by angles of the joints of the hand and elbow, by the static pressure compressing the measuring system by the hand and, also, to considerable extent, by personal varieties of hands. Hence, in this experiment, the hand and elbow joints were held at right angles and the static force was taken as about $3.5 \mathrm{~kg}$. wt.

\section{SAMPLES}

The samples used in this experiment were two types of polyvinyl chloride foam (P. V. C. foam) as described in Table 1.

Table 1. Dimension and dynamic compliance of the samples.

\begin{tabular}{c|c|c|c|c}
\hline Name & Material & $\begin{array}{l}\text { Absolute value of } \\
\text { dynamic complex } \\
\text { compliance }\left(\mathrm{cm}^{2} / \mathrm{kg}\right) \\
\text { with unit volume } \\
\text { at } 100 \mathrm{c} / \mathrm{s}\end{array}$ & Diameter & Thickness \\
\hline $\mathrm{F}_{1}$ & $\begin{array}{l}\text { P. V. C. } \\
\text { FOAM }\end{array}$ & $6 \times 10^{-2}$ & 5.0 & $\mathrm{~cm}$ \\
\hline $\mathrm{F}_{2}$ & $\begin{array}{l}\text { P.V.C. } \\
\text { FOAM }\end{array}$ & $8 \times 10^{-3}$ & 5.0 & 2.0 \\
\hline
\end{tabular}

They were taken out from the block of P. V. C. foam with the cylindrical cutter and buffed to the desired height by means of a precision buffing jig. Two types of P. V. C. foam are called $F_{1}$ and $F_{2}$ in this report. The experiment was performed at the room temperature of about $20^{\circ} \mathrm{C}$ and these samples were pressed by the hand with the constant force of about $3.5 \mathrm{~kg}$. wt.

\section{MECHANICAL MODEL}

The mechanical model approximated to the human hand was constructed to check the method of measuring the damping introduced in Eqs. 1 and 2. This model consisted of two masses of $47 \mathrm{gr}$. and $900 \mathrm{gr}$. connected alternately with two same porous elastic cylindrical materials (P. V. C. foam $F_{1}$ ), which had the diameter of $5.0 \mathrm{~cm}$. and the thickness of $3.5 \mathrm{~cm}$. The acceleration pickup was prepared in the lighter mass of $47 \mathrm{gr}$. and the one end of this model was contacted with the rigid wall as shown in Fig. 4.

The constants of this model were approximately determined by the simulator experiment of the human hand described in the next paper. However, these 


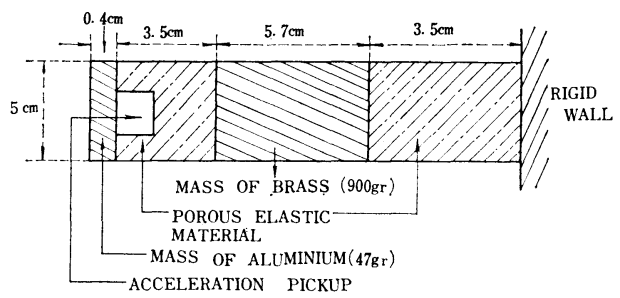

Fig. 4. Mechanical model of human hand.

constants were modified a little, since the one end of the mechanical model could not be placed in free state for the measurement of the mechanical impedance.

\section{ApParatus And Technique}

The electrical part of the measuring system of the mechanical impedance is shown schematically in Fig. 5. To measure the phase, the signals from the vibration force and acceleration pickups in the measuring system were applied to the phase meter, and the phase difference between these signals was measured, provided that both signals were adjusted to the same voltage each other. Then, to

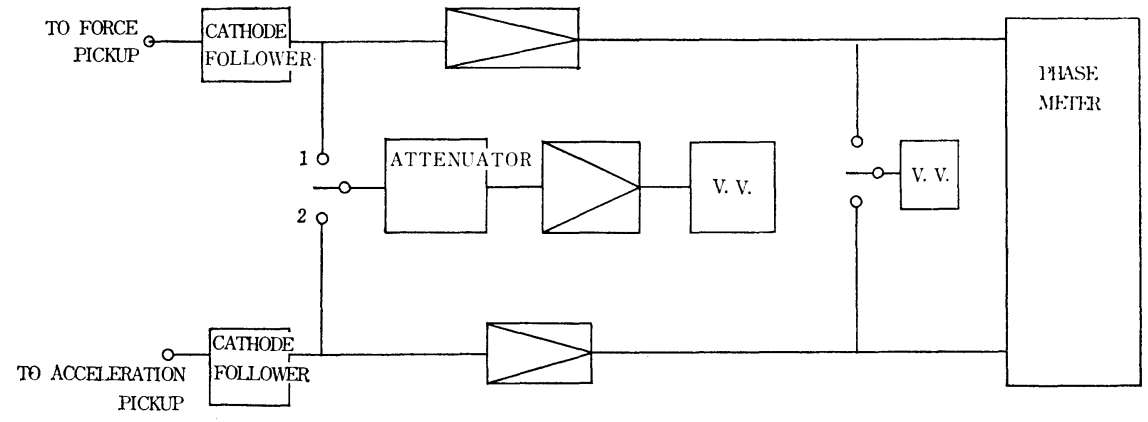

Fig. 5. Block diagram of electrical part in measuring system.

measure the magnitude of $\mathbb{H}$ in Eq. 3, the output voltage of the force pickup was adjusted to the moderate values by varying the gain of the amplifier and the switch was changed to the output terminal of the acceleration pickup without changing the gain of the electrical circuits. The attenuator was adjusted so that the vacuum tube voltmeter (V. V.) gave the same reading as before. Thus, the difference of the reading of the attenuator corresponds to the magnitude of $\mathbb{E}$.

\section{RESUlTS AND DISCUSSION}

Frequency range of the measurement in this report was limited between $20 \mathrm{c} / \mathrm{s}$ and and $500 \mathrm{c} / \mathrm{s}$, because below this range the normal sine wave could not be obtained in this vibration table and above that we could not feel the vibration.

To examine the principle of the measurement described in Eq. 7, the mechanical 


\section{STUDIES ON HAND PROTECTORS I.}

impedance of the pure mass was measured as shown in Fig. 6. The straight line indicates the calculated values and the dots in circles show the observed ones.

The result obtained coincides well with the calculated one. The unit of the impedance is expressed in the gravitational unit system in this report.

Then, the driving point mechanical impedances of the hands were measured by using three subjects (one woman and two men). In this case, the alminium disk.

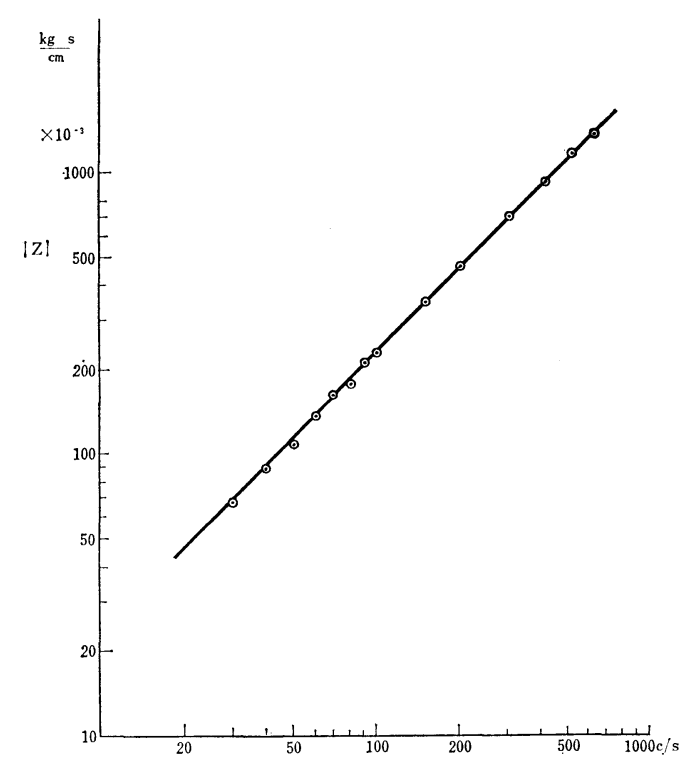

Fig. 6. Mechanical impedance of pure mass (370 gm.).

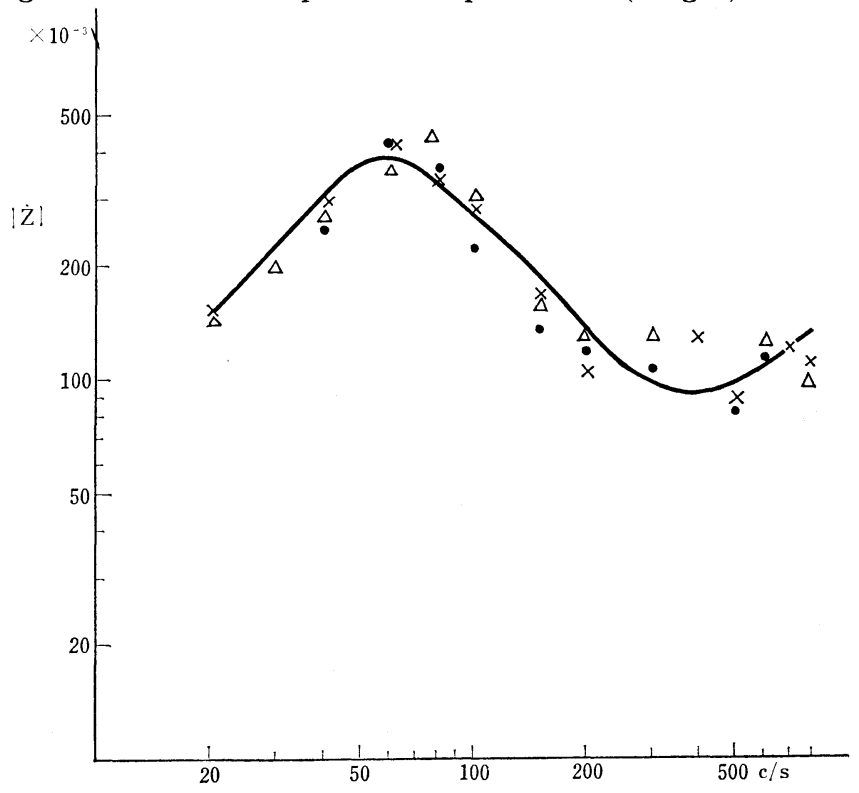

Fig. 7. Driving point mechanical impedance of hands obtained with three subjects. 


\section{T. MIWA}

should be statically compressed by the hand of the subject. When it is continued to press the measuring system constantly, the hands began to vibrate at very low frequency of about $10 \mathrm{c} / \mathrm{s}$. This phenomenon made the measurement difficult, because the false signal from the trembling of the hand was superposed on the signal from the force pickup. The measured values with three subjects are indicated in Fig. 7.

The variation from the smoothed curve seemed to be caused by the trembling of the hand described above and a variety of the human hands. From $30 \mathrm{c} / \mathrm{s}$ to 60 $\mathrm{c} / \mathrm{s}$ this result was in accord with that of Dieckmann's experiment except that the position of resonance shifted to a little higher frequency than that of his result. However, in Dieckmann's experiment he measured the mechanical impedance between $5 \mathrm{c} / \mathrm{s}$ and $70 \mathrm{c} / \mathrm{s}$. Consequently, Fig. 7 suggests that the human hand may be simulated by some mechanical system consisted of the mass, spring, and resistance possessing the resonance at about $60 \mathrm{c} / \mathrm{s}$ and the antiresonance at about $400 \mathrm{c} / \mathrm{s}$.

For. the next step, the driving point impedance of the mechanical model of the hand was measured (Fig. 8).

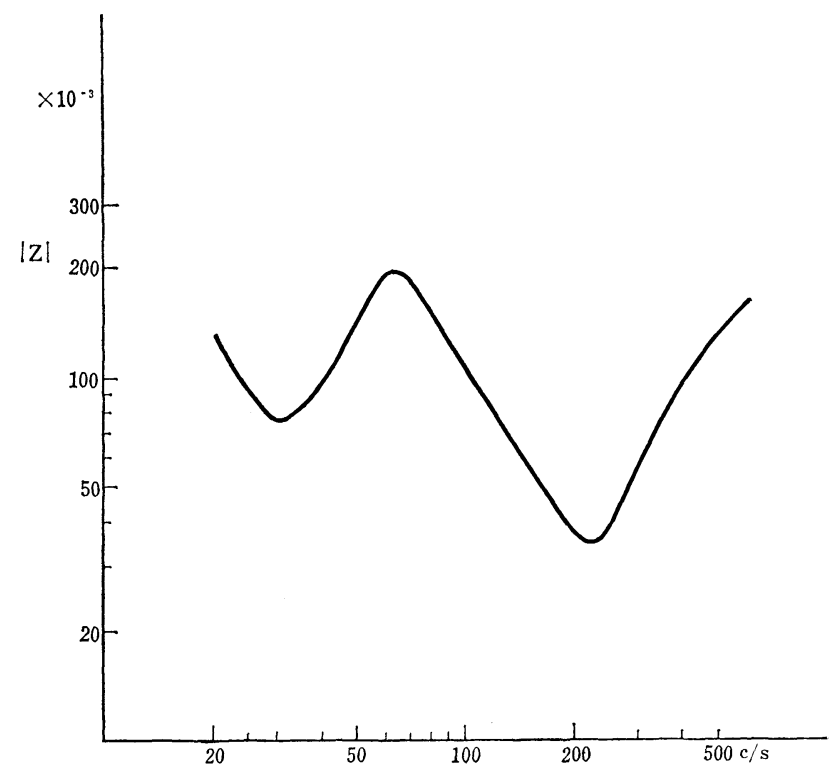

Fig. 8. Driving point mechanical impedance of mechanical model of approximated to hand.

Then, the sample $F_{1}$ was examined by employing this model. In Fig. 9, dots in circles are the values calculated from the impedance method of Eq. 2 and the curve is the observed ones from the difference between the two acceleration pickups, of which the one was at the driving point of the porous elastic sample and the other in this model. The attenuation values of this sample calculated from Eq. 2 were 


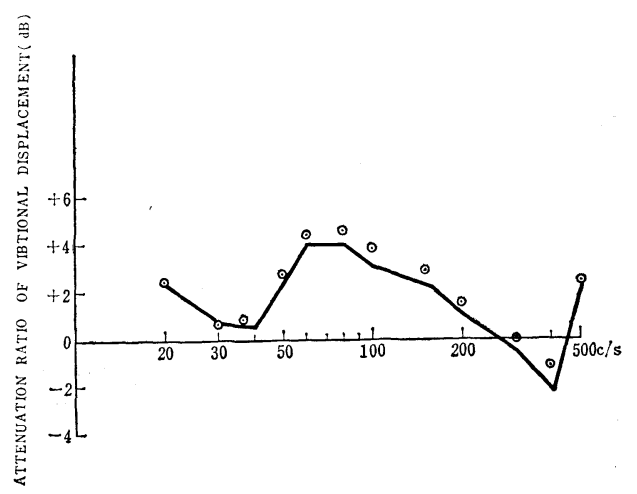

Fig. 9. Comparison between observed values from difference of two acceleration pickups (solid curve) and calculated ones from Eq. 2 (dots in circles) by using mechanical model of hand.

in good agreement with the observed ones by two pickups. Therefore, the driving point impedance method may be valid for the measurement of the protecting materials of human hands.

Finally, the driving point mechanical impedance of two samples $F_{1}$ and $F_{2}$ were measured by using the human hands of three subjects (Fig. 10 and 11). And the attenuation values of Eq. 1 calculated from Fig. 7, 10 and 11 are presented in Fig. 12.

The soild curve shows the attenuation values of the sample $F_{1}$ and the dotted curve those of the sample $F_{2}$. As the efficacy of the protection is proportional to

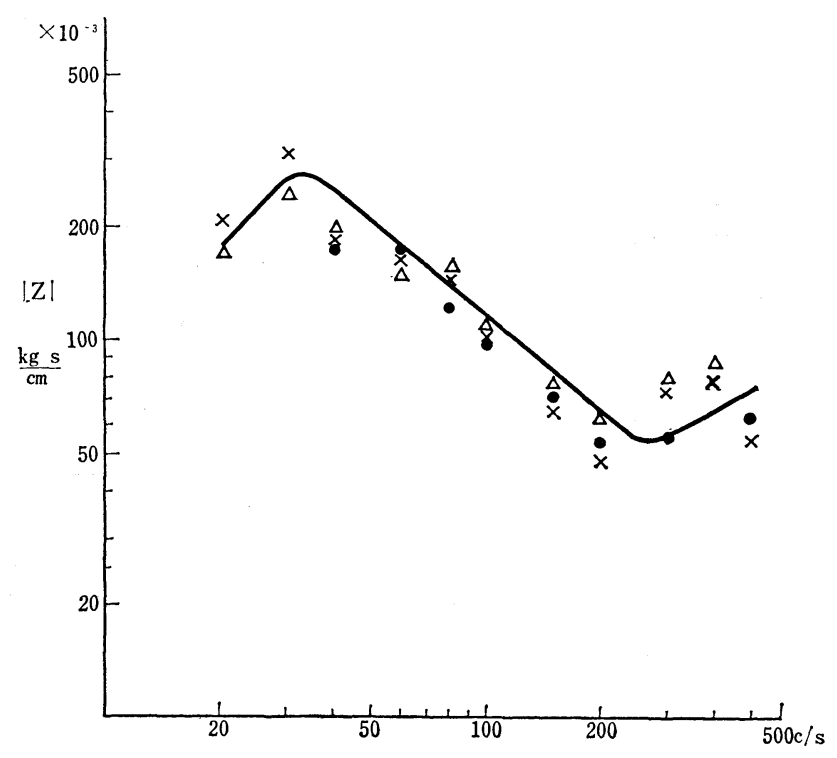

Fig. 10. Driving point mechanical impedance of sample $F_{1}$ pressed by human hands of three subjects at opposite side of driving point. 


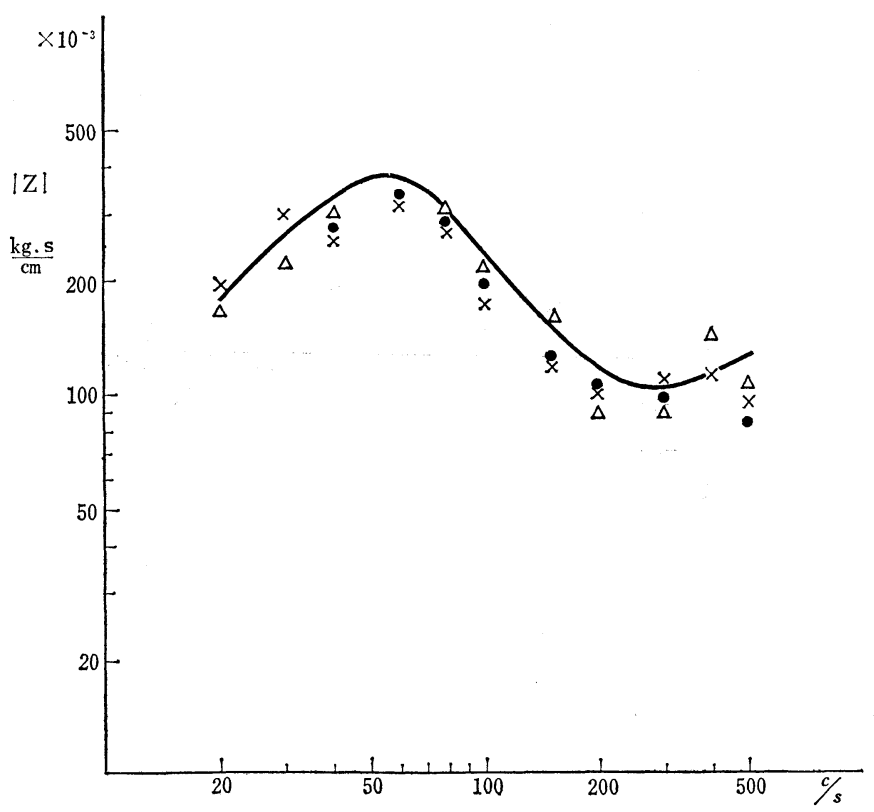

Fig. 11. Driving point mechanical impedance of sample $F_{2}$ pressed by human hands of three subjects at opposite side of driving point.

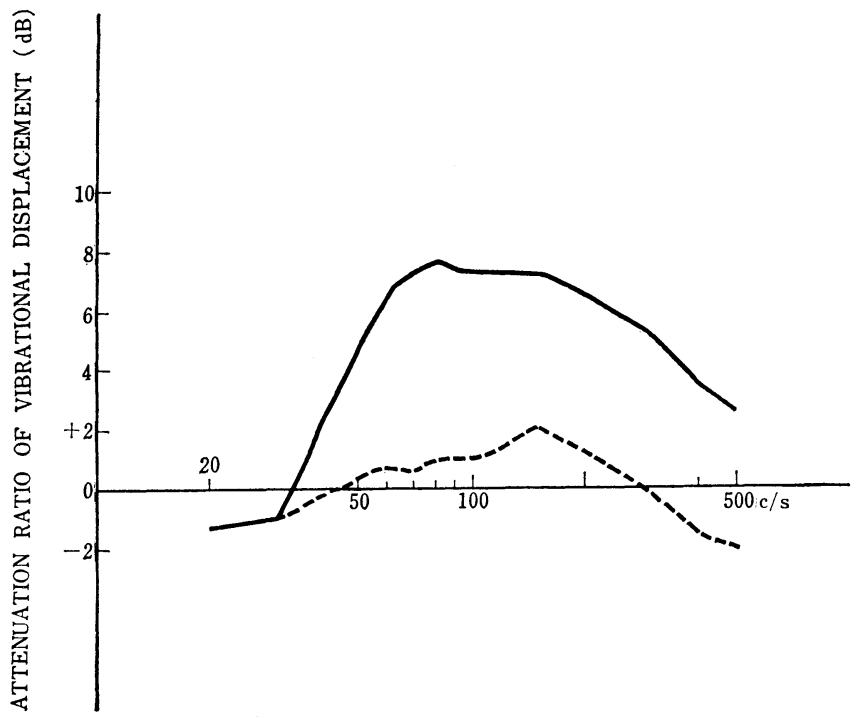

Fig. 12. Attenuation ratio of vibration displacement of sample $F_{1}$ and $F_{2}$. The solid curve indicates values of the sample $F_{1}$ and the dotted curve those of the sample $F_{2}$. 


\section{STUDIES ON HAND PROTECTORS I.}

the positive values of the attenuation ratio $(\mathrm{dB})$, the sample $F_{1}$ may be more effective by about $4 \mathrm{~dB}$ than the sample $F_{2}$ in the frequency range of $50 \mathrm{c} / \mathrm{s}$ to $300 \mathrm{c} / \mathrm{s}$. These data suggest that the efficacy may increase with their softness. However, it was recognized experimentally that the cylindrical sample of polyurethane foam (the diameter of $5.0 \mathrm{~cm}$. and the thickness of $1.5 \mathrm{~cm}$.) was inferior to the sample $F_{1}$ in the efficacy. That is, the porous elastic materials with the excessive softness or excessive hardness cannot provide the protection effect. Therefore, moderate softness must be a main factor to make the good hand protector.

From Fig. 12, it was also found that the damping efficacy of these porous elastic samples could not be obtained at the frequency range below $30 \mathrm{c} / \mathrm{s}$. Therefore, the investigation of the vibration damping at low frequencies must be the subject of future research. The influence on the protection effect due to the change of the thickness of the samples will be discussed in the next report. Based on this experiment, the effective vibration hand protectors were designed tentatively and it was proved that by using these protectors the subjects could tolerate easily the vibration above $30 \mathrm{c} / \mathrm{s}$ on the mechanical vibration table.

\section{ACKNOWLEGEMENT}

The author wishes to express his gratitude to Dr. H. Sakabe for making facilities available for carrying out the experiment and to $H$. Nakano Daiki Rubber Company for providing samples used in this work. The author is grateful for the many ingenious ideas that were offered by Dr. J. Igarashi, Proffessor of the University of Tokyo and Dr. M. Arai, Staff of Railway Technical Research Institute.

\section{REFERENCES}

1) Miura, T. (1961). J. Sci, Labour. 37. 143.

2) Harris, C. M. and Crede, C. E. (1961). Shock and Vibration Handbook, Vols. I and II. McGraw-Hill \& Co, New York.

3) Kuhn, F. (1953). Arbeitsphysiologie, 15, 79.

4) Dieckmann, D.(1958). Intern. Z. Angew. Physiol. einschl. Arbeitsphysiologie., 17, 125. 